Chapter 9

\title{
Physiologically Based Pharmacokinetic Modeling: A Tool for Understanding ADMET Properties and Extrapolating to Human
}

\author{
Micaela B. Reddy, Harvey J. Clewell III, \\ Thierry Lave and Melvin E. Andersen \\ Additional information is available at the end of the chapter \\ http://dx.doi.org/10.5772/54965
}

\section{Introduction}

Physiologically based pharmacokinetic (PBPK) models differ from classical PK models in that they include specific compartments for tissues involved in exposure, toxicity, biotransformation and clearance processes connected by blood flow (Figure 1). Compartments and blood flows are described using physiologically meaningful parameters, which allows for interspecies extrapolation by altering the physiological parameters appropriately [1]. A key benefit to PBPK models is that factors influencing the absorption, distribution, metabolism, and elimination of a compound can be incorporated into a PBPK model in a mechanistic, meaningful way, if a mechanism is understood and sufficient data are available. This mechanistic aspect is supported by physiological parameters influencing absorption (e.g., pH values and transit times through various sections of the GI tract), distribution (e.g., tissue volumes and composition), metabolism (e.g., expression levels of various hepatic enzymes and transporters involved with metabolic elimination), and elimination (e.g., glomerular filtration rate and expression levels of transporters in the kidneys involved with renal elimination), which can be explicitly incorporated in the PBPK model.

Because the models have a mechanistic basis, extrapolation to situations differing from the conditions of the data used to calibrate the model is justifiable [2]. The mechanistic basis allows PBPK models to be used to determine if results from different experimental designs are consistent, and to explore possible mechanisms responsible for unexpected or unusual data. PBPK modeling has been used to great effect for interspecies extrapolation, both among animal models [3] and for predicting human PK based on animal data [4-5]. 


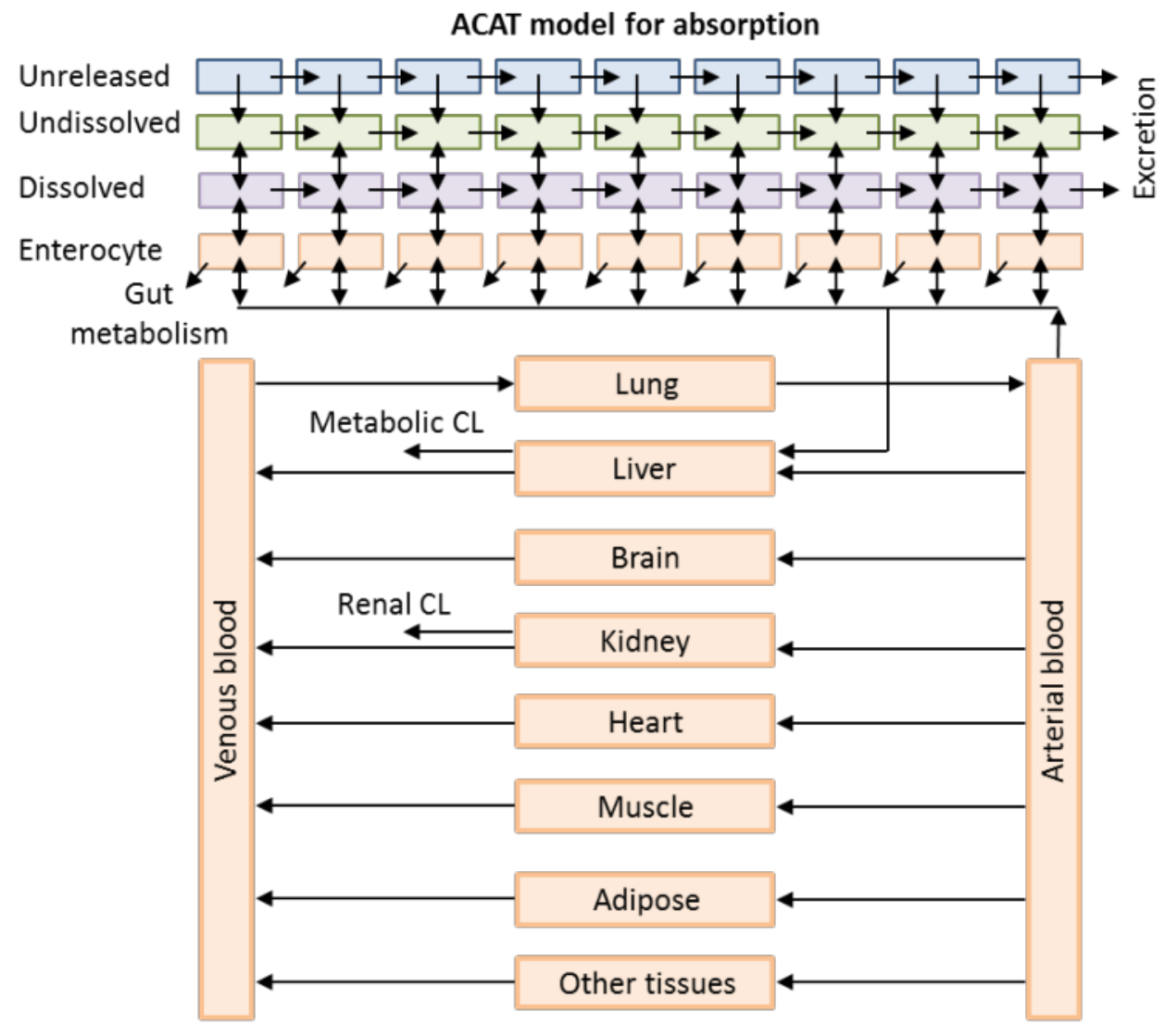

Figure 1. A schematic diagram of a PBPK model.

PBPK approaches have several advantages over other PK modeling approaches: (1) creating models from physiological, biochemical and anatomical information, entirely separate from collection of detailed concentration time-course curves; (2) evaluating mechanisms by which biological processes govern disposition of a wide range of compounds by comparison of PK results with model predictions; (3) using compounds as probes of the biological processes to gain more general information on the way biochemical characteristics govern the importance of various transport pathways in the body; (4) applying the models in safety assessments; and (5) using annotation of a modeling data-base as a repository of information on PK properties, toxicity and kinetics of specific compounds [6]. These attributes have led to widespread development of PBPK models in recent years [7], with acceleration in publication of PBPK modeling papers pertaining to drugs particularly over the last 10 years.

Here, we describe the historical development of the PBPK approach. Also, we discuss the emerging role of PBPK modeling in the pharmaceutical industry throughout drug develop- 
ment. Finally, we provide our thoughts on potential applications that have not yet been widely explored. Although advances have been made in applying PBPK modeling for biotherapeutics, this review focuses on small molecules.

\section{Historical perspective}

Interest in PK modeling in pharmacology and toxicology arises from the need to relate internal concentrations of active compounds at their target sites with the dose administered to an animal or human subject. The reason, of course, is a fundamental tenet in pharmacology or toxicology that both beneficial and adverse responses to a compound are related to the free concentration of active compound at the target tissue rather than the amount of compound at the site of absorption. The relationship between tissue dose and administered dose can be complex (Figure 2). PK models are valuable tools to assess internal dosimetry at target tissues for a wide range of exposure situations.

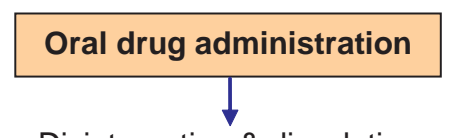

Disintegration \& dissolution

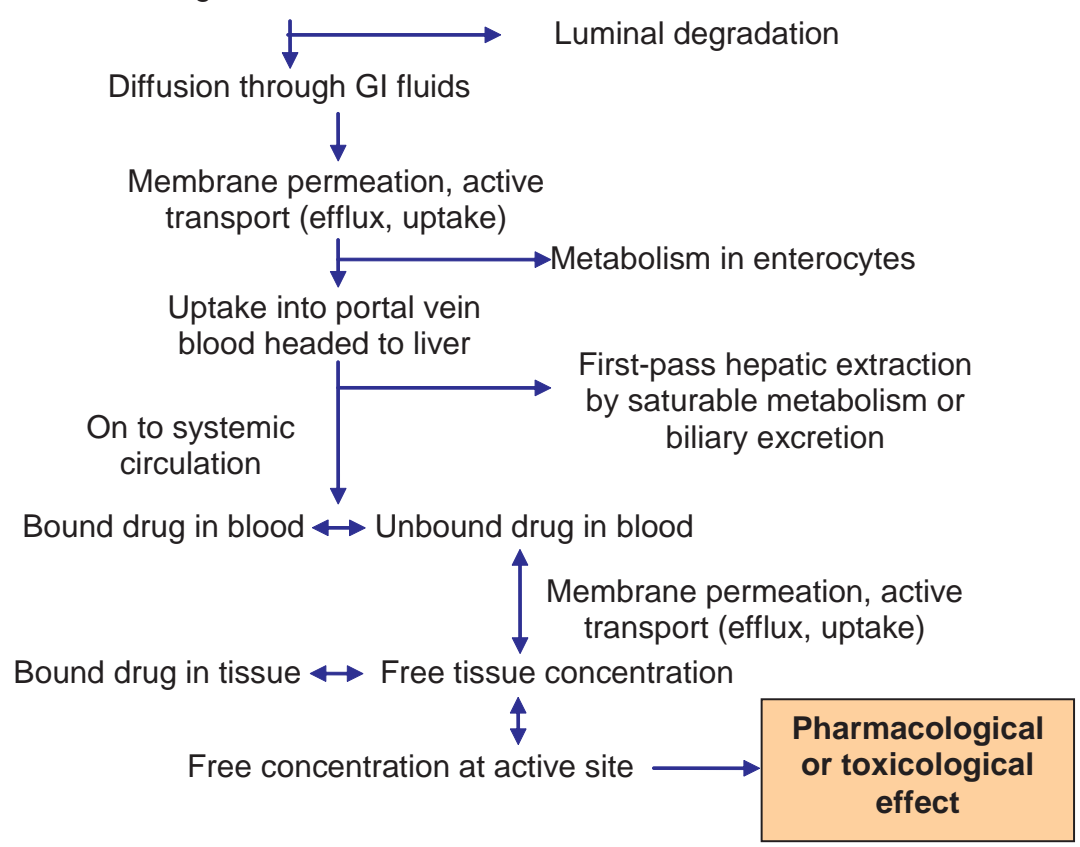

Figure 2. Aschematic diagram of the processes impacting the free concentration of drug at the target. Modified from [8]. 
In the 1930's, Teorell [9-10] provided the first physiological model for drug distribution in a set of equations for uptake, distribution, and elimination of drugs from the body. However, computational methods were not available to solve the sets of equations at this time. Exact mathematical solutions could only be obtained for simplified models in which the body was reduced to a small number of compartments that did not have direct physiological correspondence. Over the next 30 years, PK modeling focused on simpler descriptions with exact solutions rather than on models concordant with the structure and content of the biological system. These approaches are sometimes referred to as 'data-based' compartmental modeling since the work generally took the form of a detailed collection of time-course blood/excreta concentrations at various doses. Time-course curves were analyzed by assuming particular model structures and estimating a small number of model parameters by curve-fitting.

In early models, all processes for metabolism, distribution, and elimination were treated as first-order (i.e., rates changed in direct proportion to drug concentration). Two issues that particularly affected data-based PK models arose in the 1960's and early 1970's. First, increased understanding of saturation of elimination pathways led to models that were not first-order, making it difficult to derive exact solutions to the sets of equations. Second, it was realized that blood flow rather than metabolic capacity of an organ might limit clearance; blood flowlimited metabolism in an organ meant that the elimination rate could not increase indefinitely as the metabolic capacity increased [11].

Scientists trained in chemical engineering and computational methods developed PBPK models for chemotherapeutic compounds [12]. Many of these compounds are highly toxic and have therapeutic efficacy by being slightly more toxic to rapidly growing cells (cancer cells) than to normal tissues. Initial successes with methotrexate [13] led to PBPK models for other compounds, including 5-fluorouracil [14] and cisplatin [15]. These seminal contributions showed the ease with which realistic descriptions of physiology and relevant pathways of metabolism could be incorporated into PBPK models.

Although PBPK modeling was initially developed in the pharmaceutical industry [6], until recently its major use was in environmental risk assessment. In the pharmaceutical area, PBPK model use was limited mainly due to the perceived mathematical complexity of the models and the labor-intensive input data required. However, advances in the prediction of hepatic metabolism and tissue distribution from in vitro and in silico data have made the use of these models more attractive, providing the opportunity to integrate key input data from different sources to estimate PK parameters, predict plasma and tissue concentration-time profiles, and gain mechanistic insight into compound properties. Thus, interest in applying PBPK models for the discovery and development of drugs is growing [16-19].

PBPK models require physiological, physicochemical, and biochemical parameters. The physiological, mechanistic basis of the models is both their strength (the mechanistic basis provides exceptional utility) and their weakness (PBPK models can be expensive and timeconsuming to construct). However, recent contributions to the literature have demonstrated the effective application of "generic" PBPK models using the ADME data normally generated during preclinical development [5,20-22]. Since the development of the generic PBPK model- 
ing approach incorporating typical ADMET data, PBPK modeling has seen increased application in the pharmaceutical industry.

The past 10 years have seen tremendous advances in the capabilities of generic PBPK models that can simulate PK for humans or preclinical species based on a combination of physicochemical properties and in vitro data. Such generic PBPK models can be constructed using programming packages such as MATLAB®, acslX, or Berkeley Madonna. Also powerful commercial PBPK simulation tools, which incorporate useful physiologically-based absorption models in the traditional PBPK model, are now available. Such packages include, e.g., GastroPlus $^{\mathrm{TM}}$ (Simulations Plus Inc., www.simulations-plus.com), SimCyp (Simcyp, www.simcyp.com/), and PK-Sim ${ }^{\circledR}$ (Bayer Technology Services, www.pksim.com). These tools allow easy incorporation of preclinical ADMET data into a PBPK model for preclinical species and humans. The availability of such tools simplifies the technical use of PBPK models; however, a good understanding of the models and underlying equations is still mandatory in order to guarantee good interpretation of output.

Instead of taking a month or a day to construct a PBPK model, these generic models can be used to construct and validate models in a few hours, depending on the expertise of the user and the time required to gather the data. The generic PBPK model is an increasingly important tool in assessing DMPK properties in preclinical development [21-24]. PBPK models can incorporate the many complex processes that impact PK (Figure 2) in a mechanistically meaningful way (Figure 1).

\section{Current applications of PBPK modeling in drug development}

Increased application of PBPK in the pharmaceutical industry was characterized by Rowland et al., who quantified the number of scientific publications per year containing the phrase "physiologically-based pharmacokinetics" and pertaining to drugs in Web of Knowledge (Thomson Reuters); the number of publications meeting these criteria appears to be increasing exponentially over time [19]. While the extent of PBPK modeling in industry is difficult to quantify, and depends on the company, clearly PBPK modeling is increasingly used and useful, as we will illustrate in the following sections. PBPK modeling can be used from early preclinical development throughout the development process (Table 1). Drug discovery is increasingly "data rich" with high throughput chemistry generating numerous compounds that are rapidly screened for pharmacological and PK properties. Much of the typically generated preclinical ADMET data can be used in PBPK model development.

\subsection{Lead identification and optimization stages}

The first information generated includes in silico parameters (e.g., pKa, solubility, Peff, and $\log$ P values are calculated). Metabolic stability studies (e.g., in microsomes or hepatocytes) are often considered critical to determine if hepatic metabolism is a major route of elimination and if first pass metabolism might result in unacceptably low bioavailability [35,36]. Screens for permeability and solubility are often implemented early in the process. Plasma protein binding 


\begin{tabular}{|c|c|c|}
\hline Model application & Impact & Example \\
\hline \multicolumn{3}{|c|}{ Lead optimization } \\
\hline $\begin{array}{l}\text { Determining key in vitro data } \\
\text { impacting absorption and PK }\end{array}$ & $\begin{array}{l}\text { Providing guidance to chemists and providing basis for } \\
\text { screening cascade }\end{array}$ & {$[24]$} \\
\hline $\begin{array}{l}\text { Predicting PK with in silico and in } \\
\text { vitro data as inputs }\end{array}$ & $\begin{array}{l}\text { Verifying that the PK properties of a chemical series are } \\
\text { understood, prioritizing compounds for additional in vivo } \\
\text { experimentation }\end{array}$ & [21-23] \\
\hline $\begin{array}{l}\text { Understanding mechanisms } \\
\text { impacting PK }\end{array}$ & $\begin{array}{l}\text { Comparing the PBPK model to in vivo PK data to identify } \\
\text { potential reasons for mismatch and to guide additional } \\
\text { experimentation }\end{array}$ & {$[25-27]$} \\
\hline \multicolumn{3}{|c|}{ Clinical candidate selection } \\
\hline Absorption modeling & $\begin{array}{l}\text { Predicting whether a compound will exhibit a significant } \\
\qquad \text { food effect }\end{array}$ & {$[28]$} \\
\hline $\begin{array}{l}\text { Comparing exposure window } \\
\text { for lead candidates }\end{array}$ & $\begin{array}{l}\text { Linking human PK to PK/PD models for pharmacological and } \\
\text { toxicological effects improves basis for choosing best } \\
\text { candidate }\end{array}$ & {$[20]$} \\
\hline Identifying key uncertainties & Allows targeted experimentation & {$[24]$} \\
\hline \multicolumn{3}{|c|}{ Entry into human } \\
\hline $\begin{array}{l}\text { Predicting human PK and } \\
\text { efficacious dose }\end{array}$ & $\begin{array}{l}\text { Determining whether a compound will be efficacious in the } \\
\qquad \text { clinic }\end{array}$ & [5] \\
\hline $\begin{array}{l}\text { Understanding key sources of } \\
\text { variability }\end{array}$ & Provides important information for clinical trial design & {$[29]$} \\
\hline Simulate clinical trial results & $\begin{array}{l}\text { Use the PBPK model simulation to optimize clinical trial } \\
\qquad \text { design }\end{array}$ & [30-31] \\
\hline \multicolumn{3}{|c|}{ Clinical development } \\
\hline PBPK/PD modeling & $\begin{array}{c}\text { Increased understanding of dose of active compound } \\
\text { delivered to target tissues and its relationship to toxicological } \\
\text { and efficacious effects }\end{array}$ & {$[32]$} \\
\hline $\begin{array}{l}\text { Predicting PK for patient } \\
\text { population }\end{array}$ & $\begin{array}{l}\text { Incorporating physiological changes from a disease state } \\
\text { allows prediction of PK in the patient population }\end{array}$ & [33] \\
\hline $\begin{array}{l}\text { Predicting PK for population of } \\
\text { different age }\end{array}$ & $\begin{array}{l}\text { Incorporating age-specific physiological differences allows } \\
\text { prediction of PK for a specific age group, e.g., children or the } \\
\text { elderly }\end{array}$ & [34] \\
\hline
\end{tabular}

Table 1. Role of PBPK modeling at various stages in preclinical and clinical development.

might be measured to determine if a compound will have a sufficient free concentration for therapeutic efficacy [37]. The blood-to-plasma ratio might be measured aid in interpreting PK 
data [38]. PBPK models can be used to determine the key data that should be generated. For example, absorption modeling can be used to determine if solubility or permeability is likely to limit the compound's bioavailability, i.e., to determine if a solubility or permeability assay would be a useful addition to a screening cascade.

Determination of in vivo PK is more costly and slower than in vitro screening, and so using simulations to prioritize compounds for in vivo experimentation can optimize resource expenditure. PBPK can be used at an early stage to determine which compounds should go on for further experimentation [21-23]. In a study applying generic PBPK modeling to predict rat PK following iv or po administration of test compound [23], it was determined that differences in PK parameters of more than twofold could be determined based upon minimal in vitro data. But to apply the method for new series, the use of the model should be verified for several compounds before it is generally applied.

For promising compounds, a rodent PK study might be performed to determine if a compound has drug-like PK properties and adequate bioavailability. Later studies might include PK studies in a nonrodent species. Hepatocyte or microsomal clearance data can be scaled to estimate the in vivo metabolic clearance in preclinical species and humans [39-40]. The scaled clearance in preclinical species can be compared to clearance in PK studies to determine if hepatic metabolism is a major route of elimination in preclinical species. If the clearance seen in PK studies is higher than the clearance scaled from hepatocyte or microsome data, urine and/or bile might be collected in PK studies to provide additional information on mechanisms of clearance.

Because of the mechanistic basis of PBPK models, when they do not adequately describe animal PK data, this means that a biological phenomenon affecting PK has not been included in the model and is not represented by the assays used to screen the compounds. Therefore, if a PK issue for a promising compound becomes apparent, PBPK modeling allows you to determine which mechanisms are consistent with the observed data. This information can be used to guide further experimentation to arrive more rapidly at the desired information. For example, Peters [25-26] proposed a method for assessing "lineshape" mismatch between simulated and observed oral profiles to gain mechanistic insights into processes impacting absorption and PK (e.g., saturable metabolism, enterohepatic cycling, transporter involvement in absorption from the gut, and regional variation in gut absorption). In related work, Peters and Hultin [27] used a similar approach to identify drug-induced delays in gastric emptying.

Preclinical ADMET studies provide pharmaceutical scientists with quantitative information on the PK in preclinical species and qualitative information on the potential human PK behavior of a compound. Using PBPK modeling, the results of the various preclinical assays can be integrated to provide a quantitative prediction of human PK $[5,41]$. Once the PBPK model exists, it can be used to determine which compounds have the largest impact on PK, providing chemists with additional clear information for the development of improved compounds.

During preclinical development, ADMET data is constantly being generated. A PBPK model can act as a repository of current information, and is easily updated when new PK or PD data 
become available. But adequate data are necessary to apply PBPK, which can be a limitation. For example, if a company does not generate hepatocyte or microsome data that can be scaled for a reasonably accurate estimate of metabolic clearance, the utility of PBPK is reduced.

\subsection{Clinical candidate selection}

Once PO PK data and detailed pharmaceutical data are available, physiologically based absorption modeling can be useful to verify that factors impacting oral absorption are understood and to help guide formulation development. Data on solubility, dissolution, precipitation, membrane permeation, whether a compound is a transporter substrate, and metabolic stability (particularly for CYP3A4, but other enzymes are also expressed in enterocytes [42]) are all important for predicting absorption [43]. PBPK models incorporate physiological information on the GI tract (e.g., transit times, $\mathrm{pH}$ values and transit times in the various regions). However, it can be important to carefully consider whether typical physiological conditions are appropriate for a given absorption simulation. For example, in typical dogs administered a solution, the stomach $\mathrm{pH}$ might be over 7 , but in pentagastrin-pretreated dogs the stomach $\mathrm{pH}$ might be less than 3 for a period of time [44].

Absorption modeling can be very useful at later stages, e.g., for predicting whether PK will be different when a compound is administered with food. Jones et al. [28] developed GastroPlus human oral absorption models for six compounds. Food effects were predicted for a range of doses and compared to the results from human food effect studies. In general, the models were able to predict whether a food effect would be major (i.e., for two compounds) or minor (i.e., for four compounds).

PBPK can assist with clinical candidate selection where numerous factors must be considered and data related to the PK and PD of a compound need to be combined and compared in a rational way. Parrott et al. [20] demonstrated the use of PBPK modeling to select the best compound from among five candidates for the clinical lead. The preclinical data for each candidate was integrated and the efficacious human doses and associated plasma exposures were estimated. The PBPK models were linked to an Emax PD model so that the dose resulting in a $90 \%$ effect could be identified. This example showed that the PBPK approach facilitates a sound decision on the selection of the optimal molecule to be progressed by integrating the available information and focusing the attention onto the expected properties in human. Importantly, the method can include estimates of variability and uncertainty in the predictions to verify that decisions are based on significant differences between compounds.

\subsection{Supporting entry into humans}

After a clinical candidate has been selected, the first-in-human (FIH) dose must be selected. One method for selecting the FIH dose is to use a human PK model (developed based on preclinical ADMET data) to determine the dose that will result in a systemic exposure identified as therapeutic based on preclinical pharmacology data [45]. Determining the exposure required for efficacy requires a good understanding of the PK/PD relationship and what is driving the PD, be it Cmin, Cmax, AUC, or time above a threshold concentration. 
Regardless of what is driving the PD, a human PBPK model can be used to determine the dose and regimen that can meet the efficacy requirement.

Luttringer et al. [41] examined the ability of PBPK modeling to predict human PK using epiroprim as a test compound due to significant species differences in its PK properties. By incorporating information on species differences in PK properties from in vitro studies (e.g., in protein binding, the blood-to-plasma ratio, and intrinsic clearance in hepatocytes), PBPK modeling was able to reduce the uncertainty inherent in interspecies extrapolation and to provide a better prediction of human PK than allometric scaling or by direct scaling of hepatocyte data.

Recently, Jones et al. [5] reported a method for predicting human PK based on preclinical data by separately predicting absorption, distribution and elimination from a physiological perspective. Human PK of 19 compounds that had entered into humans was predicted using Dedrick plot analysis (i.e., a type of allometric scaling of concentration-time profiles) and PBPK modeling. The ability of PBPK modeling to predict PK in preclinical species was tested, and the human PK was only predicted if the model could predict PK in preclinical species. Based on this criterion, for $70 \%$ of the 19 compounds included in the study a human PK prediction could be made. The prediction accuracy using PBPK was good for oral clearance, volume of distribution, terminal elimination half-life, Cmax, AUC, and tmax. The human PK data were more accurately predicted by the PBPK modeling approach than by the empirical approach. The strategy proposed by Jones et al. [5] can guide the strategy for gathering the data necessary for a complete understanding of likely human PK behavior.

Poor predictions with PBPK models are often a result of incomplete knowledge resulting in processes not correctly incorporated into the model (e.g., for biliary clearance and enterohepatic recirculation there are limited options for quantitatively predicting the effects on human PK). For such situations, an alternative method of performing the human PK extrapolation (e.g., allometric scaling) might seem attractive. However, allometric scaling is based on the assumption that clearance is proportional to $\mathrm{BW}^{0.75}$ and that steady-state volume of distribution is proportional to $\mathrm{BW}^{1}$ due to physiological properties (i.e., the same physiological properties that are incorporated in PBPK models). If PBPK modeling does not appear to be a good method for a compound, there is no reason to believe that allometric scaling or other empirical methods will work either. Additionally, developing methods for including increasingly complex PK mechanisms (e.g., first-pass metabolism and transporters in the gut, EH cycling, biliary excretion, impact of transporters on tissue concentration and elimination, and multiple pathways of elimination) is an active area of research, and methods for predicting human PK even with PK complications is increasingly possible.

In cases where uncertainty is high, the PBPK model can be a valuable tool for determining appropriate experiments and simulating "what-if" scenarios. Uncertainty can be illustrated by presenting a range of results (e.g., AUC, Cmax, Cmin, or complete time-course concentrations) for a range of values of a key parameter that is not known with great certainty. If uncertainty is too great, additional experiments can be designed to aid in narrowing the possible values of key parameters. PBPK modeling can be used as a tool to understand the 
quantitative impact of uncertainty from key knowledge gaps, and also helps to minimize uncertainty due to species differences in PK properties.

\subsection{Clinical development}

Population PK approaches and nonlinear mixed effect modeling are the techniques most used for analysis of clinical data. The Bayesian population PK approach can also be applied with PBPK models [46-47]. But this alternative has not been adopted by the pharmaceutical industry. Although several useful clinical applications of PBPK models have been demonstrated in the literature including the following examples, there are many potentially valuable applications that remain to be exploited [48].

PBPK simulations can be used to optimize clinical design. Chenel et al. [30] recently demonstrated selecting time points for an optimized sparse sampling design for a DDI clinical trial using Monte Carlo simulations for the victim midazolam and the CYP3A4 inhibitor that was the test compound. The PBPK models were parameterized using only in vitro data. The PBPK predictions of PK for both compounds were good using the PBPK model, but for the test compound variability was overestimated somewhat [32]. Regardless, data from the optimized trial design resulted in similar CL/F estimates and the same conclusion as the full empirical design upon analysis using a population PK approach.

Blesch et al. [32] demonstrated how PBPK modeling could fit into a clinical modeling strategy for capecitabine, a triple prodrug of 5-fluorouracil. For capecitabine, the modeling strategy included nonlinear mixed-effect modeling and PK/PD modeling for safety and efficacy to analyze clinical data. Nevertheless, a simple PBPK model incorporating the GI tract, blood, liver, tumor, and non-eliminating tissues compartments describing the PK of capecitabine and three metabolites including 5-fluorouracil was still useful for drug development. This approach allowed mechanistic knowledge gained preclinically to be leveraged. The PBPK model provided a tool for understanding the relationship between the dose of the triple prodrug capecitabine and the delivery of active compound to the tumor.

Because of the heterogeneity of the human population, it is expected that there will be a broad range of responses to biological effects of drugs. This heterogeneity is produced by interindividual variations in physiology, biochemistry, and molecular biology, reflecting both genetic and environmental factors, and results in differences among individuals in PK and PD. Because the parameters in a PBPK model have a direct biological correspondence, they provide a useful framework for determining the impact of observed variations in physiological and biochemical factors on the population variability in dosimetry. Willman et al. [49] demonstrated an approach for generically incorporating interindividual differences in physiological parameters using PK-Sim and showed that for two drugs, ciproflaxin and paclitaxel, the predicted variability was close to that observed clinically. Predicting variability in PK from factors including genetics (e.g., polymorphisms in enzymes that metabolize drugs), disease state (e.g., hepatic impairment or impaired renal function), and age from pediatric to elderly patients is a useful application of PBPK [19]. 
Recently there has been considerable work ongoing to predict PK of drugs in the pediatric population using PBPK modeling. For such simulations, the PBPK model must incorporate age-specific differences in body weight, organ weights, blood flows to tissue compartments, compartment volumes, plasma protein binding, renal function, and hepatic P450 expression levels, among other factors [50-51]. A recent study demonstrated successfully predicting PK in children for acetaminophen, alfentanil, morphine, theophylline, and levofloxacin, based on existing PBPK models for adults verified and/or calibrated using adult PK data [34]. This approach has utility in designing clinical trials for the first time dosing in children [50]. PBPK modeling can also be applied to predict PK in infants and neonates, e.g., as was done for oseltamivir [52]. PBPK modeling has been proposed as a useful tool benefitting the learn-and-confirm paradigm in pediatric trials and improving pediatric drug development programs [53].

The mechanistic basis of PBPK modeling also allows the modification of physiological parameters to describe disease state. For example, the physiological changes with cirrhosis of the liver include alterations in hepatic P450 expression and liver size, albumin and $\alpha-1$ acid glycoprotein, blood flows, and renal function [54]. These physiological differences can be specifically incorporated in the PBPK model, allowing the prediction of PK in the population of people suffering from cirrhosis of the liver. The ability to predict PK for lidocaine and alfentanil for patients with differing degrees of cirrhosis was recently demonstrated by Edginton and Willmann [33] with promising results.

\subsection{Support for regulatory decision making}

Recent publications and regulatory guidance documents indicate that PBPK modeling is becoming increasingly useful from a regulatory perspective. The EMA has indicated that PBPK modeling can be a useful tool for the clinical investigation of hepatic impairment on pharmacokinetics [55]. Both the FDA and EMA in their drug-drug interaction (DDI) guidance documents mention PBPK modeling as a useful tool, e.g., for supporting DDI understanding and for designing DDI studies, and potentially for supporting labeling [56-57]. The FDA experience with applications of PBPK modeling during regulatory review [58-59] and pediatric drug trials [53] have been described. Clinical pharmacology reviewers at the FDA have done PBPK modeling to address PK and/or DDI issues in cases where the sponsor did not [58].

Guidance on best practices for PBPK modeling in addressing regulatory questions has been offered [60]. The successful application of PBPK modeling requires sufficient data and understanding of key processes impacting PK. For example, for using PBPK modeling to understand and simulate DDIs, information is needed on the mechanism of elimination and the fraction of compound metabolized through various pathways (e.g., mediated by different P450s). It has been recommended that a human, in vivo mass balance study be completed for the greatest confidence in DDI simulations [58]. To use PBPK modeling to address a regulatory issue, not only must sufficient data be available, but the effect of physiology on PK for a given issue must be understood. Potential data gaps such as a lack of understanding of developmentrelated and disease-related effects on physiology and PK can limit application of PBPK in certain situations. 
An early example of the application of PBPK in regulatory decision making is the case of a safety assessment for retinoic acid [61]. In the early 1990s all-trans retinoic acid (ATRA) was being considered for marketing approval by the Food and Drug Administration (FDA) for the indication of photo-damaged skin (wrinkles). The director of the FDA Center for Drug Evaluation and Research (CDER) requested the sponsor to evaluate, using PBPK simulation, the potential fetal exposure to ATRA applied topically in women of reproductive age. Aware that ATRA, like its isomer 13-cis retinoic acid, is highly teratogenic and that up to $10 \%$ of a topically applied dosage is absorbed systemically, FDA sought reassurance that significant fetal exposure and teratogenic effect potential would not result during clinical use. PBPK simulation was considered the only rational and ethical method of risk assessment available. The sponsor supported a PBPK analysis [4] that provided the necessary assurance to the FDA during its review and subsequent approval. FDA encourages sponsors to adopt PBPK, when appropriate and depending on the questions, during drug development with the aim to facilitate and enhance the capability to make better predictions, improve understanding, and provide improved regulatory decision making [61].

\section{Future directions}

\subsection{Interindividual variability}

As described in a previous section, investigators have begun using PBPK to understand variability from several sources: (1) variations across a population of healthy adults from physiological differences (e.g., body weight or sex) [62] or genetic polymorphisms [63]; (2) variations across a population from age differences, e.g., infants or the elderly [64-65]; and (3) variations from health status (e.g., from differences in protein or enzyme expression, tissue blood flow, or tissue compartment volumes or function altered by disease state) [33]. To the extent that the variation in physiological and biochemical parameters across these population dimensions can be elucidated, PBPK models can be used together with Monte Carlo uncertainty analysis to integrate their effects on the in vivo kinetics of a compound and predict the resulting impact on the distribution of PK across the population. The use of Monte Carlo uncertainty analysis has been described for drugs [66-67], but the ability of this powerful approach to evaluate the impact of interindividual variability on clinical trials outcomes is not yet fully utilized in the pharmaceutical industry.

There has been a tendency in drug development to use information on the variability of a specific parameter, such as the in vitro activity of a particular enzyme, as the basis for expectations regarding the variability in dosimetry for in vivo exposures. However, whether or not the variation in a particular physiological or biochemical parameter will have a significant impact on in vivo dosimetry is a complex function of interacting factors. In particular, the structures of physiological and biochemical systems frequently involve parallel processes (e.g., blood flows, metabolic pathways, excretion processes), leading to compensation for the variation in a single factor. Moreover, physiological constraints may limit the in vivo impact of variability observed in vitro. For instance, high affinity intrinsic clearance can result in 
essentially complete metabolism of all the compound reaching the liver in the blood; under these conditions, variability in amount metabolized in vivo would be more a function of variability in liver blood flow than variability in metabolism in vitro. Thus it is often true that the whole (the in vivo variability in dosimetry) is less than the sum of its parts (the variability in each of the PK factors). Due to the complex interactions among factors impacting PK, speculation regarding the extent of population variability on the basis of the observed variation in a single factor can be highly misleading. This possibility has been illustrated in the case of the impact of the CYP2C9 polymorphism on warfarin kinetics [63].

\subsection{Modeling of pharmacodynamics}

The growing popularity of the PBPK modeling approach represents a movement from simpler kinetic models toward more biologically realistic descriptions of the determinants that regulate disposition of drugs in the body. To a large extent, the application of these PBPK models to study the time courses of compounds in the body is simply an integrated systems approach to understanding the biological processes that regulate the delivery of drugs to target sites. Many PBPK models integrate information across multiple levels of organization, especially when describing interactions of compounds with molecular targets, such as reversible binding of ligands to specific receptors, as in the case of methotrexate binding to dihydrofolate reductase $[13,68]$. In such cases, the PBPK models integrate molecular, cellular, organ level, and organism-level processes to account for the time courses of compounds, metabolites, and bound complexes within organs and tissues in the body.

While the goal in applying a PBPK model is to predict plasma and tissue concentrations of a drug, the overall goal of using PBPK modeling in efficacy and safety assessment with drugs is broader. PBPK models once developed are extensible. The goal in the larger context is to understand the relationship between dose administered, the dose reaching the active site, and the resulting biological response. The specific steps that lead from these dose metrics to tissue, organ and organism-level responses, have usually been considered part of the PD process. In general, PD models used in drug evaluation have been more empirical, utilizing simple effect compartments correlated with blood or tissue concentrations of active compound. Inexorably, the systems approach will advance into the PBPK/PD and physiologically based pharmacodynamics (PBPD) arena, i.e., into a systems biology approach for describing perturbations of biological systems by compounds and the exposure/dose conditions under which these perturbations become sufficiently large to pose significant health risks or to achieve specific therapeutic outcomes. In fact, PBPK/PD models of toxic effects have been demonstrated to be useful for risk assessment purposes, as in the case of a PBPK/PD model linking chloroform metabolism, reparable cell damage, cell death, and regenerative cellular proliferation [69].

The systems biology approach focuses on normal biological function and the perturbations associated with exposure to compounds. Perturbations of biological processes by compounds lead to either adverse responses (toxicity) or restoration of normal function to a compromised tissue (efficacy). The effects of compounds, whether for good or ill, can best be described by PBPK approaches linked through PBPD models of responses of cellular signaling networks. Toxicity and efficacy are then defined by an intersection of compound action with the biolog- 
ical system. Toxicology and pharmacology are disciplines at the interface of chemistry/ pharmacokinetics and biology/pharmacodynamics. Clearly, the main differences in the next generation of systems approaches in PK and PD modeling will be the increasingly detailed descriptions of biology afforded by new technologies and the expansion of modeling tools available for describing the effects of compounds on biological signaling processes.

Of particular importance, in using a PBPK model the pharmacodynamic effects of a drug can be investigated more directly, relating the effects to free concentration in the tissue (e.g., the brain) where the compound interacts with the biological system, rather than attempting to elucidate a potentially indirect relationship with plasma concentrations. By obtaining quantitative information on the dose-responses for both the efficacy and toxicity of the compound, the PBPK model can be exercised to evaluate the potential to increase the efficacy/toxicity ratio of the drug through manipulation of dose and route using novel drug delivery systems. These and other attributes of PBPK models for organizing and interpreting diverse data sets, with the specific goals of understanding efficacy and toxicity, are reviving interest in applying these tools in drug development and evaluation [32].

The rapid development of computational chemistry [70], genomics [71], and high-throughput screening [72] has brought increasing attention to the discovery phase of drug development, including growing interest in "discovery toxicology" [73]. PBPK modeling can play a complementary role to two other technologies that are finding increasing use in drug discovery: QSAR analysis and genomics. QSAR can be used to estimate compound-specific parameters for the PBPK model, while genomic data can provide mode of action insights that drive model structure decisions such as the selection of the appropriate dose metric and its linkage to pharmacodynamic elements. The PBPK model provides a quantitative biological framework for integrating the physicochemical characteristics of the drug candidate, together with in vitro data on its ADME and toxicity, within the constraints of the fundamental physiological and biochemical processes governing compound behavior in vivo. This approach is particularly effective when used consistently during drug development, because the information gained from modeling of previous candidate compounds can greatly facilitate model development for new compounds with similar structures or properties.

\section{Conclusions}

PBPK modeling has been identified as a technology that can be used by the pharmaceutical industry to accelerate the drug development process [17,62,74-75]. Conveniently, ADMET data typically generated during preclinical development can be used to develop PBPK models. PBPK models allow better use of these data by serving as a structured repository for quantitative information on the compound, a conceptual framework for hypothesis testing, and a quantitative platform for prediction. The power of PBPK modeling for understanding properties underlying PK and for allowing uncertainty and variability analysis make this tool valuable to the pharmaceutical industry. 


\section{Author details}

Micaela B. Reddy ${ }^{1}$, Harvey J. Clewell $\mathrm{III}^{2}$, Thierry Lave ${ }^{3}$ and Melvin E. Andersen ${ }^{2}$

*Address all correspondence to: HClewell@theHamner.org

1 Hoffmann-La Roche Inc., Nutley, New Jersey, USA

2 The Hamner Institutes for Health Sciences, Research Triangle Park, North Carolina, USA

3 Hoffmann-La Roche Inc., Basel, Switzerland

\section{References}

[1] Clewell HJ III, Reddy MB, Lave T, Andersen ME. Physiologically Based Pharmacokinetic Modeling. In: Gad SC. (ed.) Preclinical Drug Development Handbook. Hoboken: John Wiley \& Sons; 2008. p. 1167-1227.

[2] Aarons L. Editors' view. Physiologically based pharmacokinetic modeling: a sound mechanistic basis is needed. British J Clin Pharm 2005;60(6) 581-583.

[3] Dedrick RL, Bischoff KB. Species similarities in pharmacokinetics. Fed Proc 1980;39: 54-59.

[4] Clewell HJ, Andersen ME, Wills RJ, Latriano L. A physiologically based pharmacokinetic model for retinoic acid and its metabolites. J Am Acad Dermatol 1997;36: S77-85.

[5] Jones HM, Parrott N, Jorga K, Lave T. A novel strategy for physiologically based predictions of human pharmacokinetics. Clin Pharmacokinet 2006;45: 511-542.

[6] Andersen ME, Yang RSH, Clewell HJ, Reddy MB. Introduction: A Historical Perspective of the Development and Application of PBPK Models. In: Reddy MB, Yang RSH, Clewell HJ III, Andersen ME. (eds.) Physiologically Based Pharmacokinetic Modeling: Science and Applications. Hoboken: John Wiley \& Sons; 2005. p. 1-18.

[7] Reddy MB, Yang RSH, Clewell HJ III, Andersen ME, editors. Physiologically Based Pharmacokinetic Modeling: Science and Applications. Hoboken: John Wiley \& Sons; 2005.

[8] Martinez MN, Amidon GL. A mechanistic approach to understanding the factors affecting drug absorption: A review of fundamentals. J Clin Pharmacol 2002;42: $620-643$.

[9] Teorell T. Kinetics of distribution of substances administered to the body. I. The extravascular mode of administration. Arch Int Pharmacodyn 1937;57: 205-225. 
[10] Teorell T. Kinetics of distribution of substances administered to the body. I. The intravascular mode of administration. Arch Int Pharmacodyn 1937;57: 226-240.

[11] Andersen ME. Saturable metabolism and its relation to toxicity. Crit Rev Toxicol 1981;9:105-150.

[12] Bischoff KB, Brown RG. Drug distribution in mammals. Chem Eng Prog Symp Ser 1966;62(66): 33-45.

[13] Bischoff KB, Dedrick RL, Zaharko DS, Longstreth, JA. Methotrexate pharmacokinetics. J Pharm Sci 1971;60: 1128-1133.

[14] Collins JM, Dedrick RL, Flessner MF, Guarino AM. Concentration dependent disappearance of fluorouracil from peritoneal fluid in the rat: Experimental observations and distributed modeling. J Pharm Sci 1982;71: 735-738.

[15] Farris FF, Dedrick RL, King FG. Cisplatin pharmacokinetics: Applications of a physiological model. Toxicol Lett 1988;43, 117-137.

[16] Lupfert C, Reichel A. Development and application of physiologically based pharmacokinetic-modeling tools to support drug discovery. Chem \& Biodiversity 2005;2: 1462-1486.

[17] Nestorov I. Whole body pharmacokinetic models. Clin Pharmacokinet 2003;42: 883-908.

[18] Lave T, Parrott N, Grimm HP, Fleury A, Reddy M. Challenges and opportunities with modelling and simulation in drug discovery and drug development. Xenobiotica 2007;37(10-11): 1295-1310.

[19] Rowland M, Peck C, Tucker G. (2011). Physiologically-based pharmacokinetics in drug development and regulatory science. Cho AK (ed.). Book series, Annual Review of Pharmacology and Toxicology, vol 51, pp. 45-73.

[20] Parrott N, Jones H, Paquereau N, Lave T. Application of full physiological models for pharmaceutical drug candidate selection and extrapolation of pharmacokinetics to man. Basic \& Clin Pharmacol \& Toxicol 2005;96: 193-199.

[21] Brightman FA, Leahy DE, Searle GE, Thomas, S. Application of a generic physiologically based pharmacokinetic model to the estimation of xenobiotic levels in rat plasma. Drug Metab Disposition 2006;34: 84-93.

[22] Brightman FA, Leahy DE, Searle GE, Thomas S. Application of a generic physiologically based pharmacokinetic model to the estimation of xenobiotic levels in human plasma. Drug Metab Disposition 2006;34: 94-101.

[23] Parrott N, Paquereau N, Coassolo P, Lave T. An evaluation of the utility of physiologically based models of pharmacokinetics in early drug discovery. J Pharm Sci 2005;94: 2327-2343. 
[24] Berry P, Parrott N, Reddy M, David-Pierson P, Lavé T. Putting it all together. Faller B, Urban L. (eds.) In: Hit and Lead Profiling: Identification and Optimization of Drug-like Molecules. In book series, Methods and Principles in Medicinal Chemistry. Series edited by R Mannhold, H Kubinyi and G Folkers. Hoboken: John Wiley \& Sons; 2010. p. 221-242.

[25] Peters S. Evaluation of a generic physiologically based pharmacokinetic model for lineshape analysis. Clin Pharmacokinet 2008;47: 261-275.

[26] Peters SA. Identification of intestinal loss of a drug through physiologically based pharmacokinetic simulation of plasma concentration-time profiles. Clin Pharmacokinet 2008;47(4): 245-259.

[27] Peters SA, Hultin L. Early identification of drug-induced impairment of gastric emptying through physiologically based pharmacokinetic (PBPK) simulation of plasma concentration-time profiles in rat. J Pharmacokinet Pharmacodynam 2008;35(1): 1-30.

[28] Jones HM, Parrott N, Ohlenbusch G, Lavé T. Predicting pharmacokinetic food effects using biorelevant solubility media and physiologically based modelling. Clin Pharmacokinet 2006;45: 1213-1226.

[29] Jamei M, Dickinson GL, Rostami-Hodjegan A. A framework for assessing inter-individual variability in pharmacokinetics using virtual human populations and integrating general knowledge of physical chemistry, biology, anatomy, physiology and genetics: A tale of 'bottom-up' vs 'top-down' recognition of covariates. Drug Metab Pharmacokinet 2009;24(1): 53-75.

[30] Chenel M, Bouzom F, Aarons L, Ogungbenro K. Drug-drug interaction predictions with PBPK models and optimal multiresponse sampling time designs: application to midazolam and a phase I compound. Part 1: comparison of uniresponse and multiresponse designs using PopDes. J Pharmacokinet Pharmacodynam 2008;35(6): 635-659.

[31] Chenel M, Bouzom F, Cazade F, Ogungbenro K, Aarons L, Mentre F. Drug-drug interaction predictions with PBPK models and optimal multiresponse sampling time designs: application to midazolam and a phase I compound. Part 2: clinical trial results. J Pharmacokinet Pharmacodynam 2008;35(6): 661-681.

[32] Blesch KS, Gieschke R, Tsukamoto Y, Reigner BG, Burger HU, Steimer JL. Clinical pharmacokinetic/pharmacodynamic and physiologically based pharmacokinetic modeling in new drug development: the capecitabine experience. Invest New Drugs 2003;21(2): 195-223.

[33] Edginton AN, Willmann S. Physiology-based simulations of a pathological condition prediction of pharmacokinetics in patients with liver cirrhosis. Clin Pharmacokin 2008;47(11): 743-752. 
[34] Edginton AN, Schmitt W, Willmann S. Development and evaluation of a generic physiologically based pharmacokinetic model for children. Clin Pharmacokin 2006;45(10): 1013-1034.

[35] Lave T, Dupin S, Schmitt C, Valles B, Ubeaud G, Chou RC, Jaeck D, Coassolo P. The use of human hepatocytes to select compounds based on their expected hepatic extraction ratios in humans. Pharm Res 1997;14: 152-155.

[36] Thompson,TN. Early ADME in support of drug discovery: The role of metabolic stability studies. Current Drug Metabolism 2000;1: 215-241.

[37] Poggesi I. Predicting human pharmacokinetics from preclinical data. Current Opinion in Drug Discovery \& Development 2004;7: 100-111.

[38] Hinderling PH. Red blood cells: A neglected compartment in pharmacokinetics and pharmacodynamics. Pharmacol Rev 1997;49: 279-295.

[39] Riley RJ, McGinnity DF, Austin RP. A unified model for predicting human hepatic, metabolic clearance from in vitro intrinsic clearance data in hepatocytes and microsomes. Drug Metab Disposition 2005;33: 1304-1311.

[40] Obach RS. Prediction of human clearance of twenty-nine drugs from hepatic microsomal intrinsic clearance data: An examination of in vitro half-life approach and nonspecific binding to microsomes. Drug Metab Disposition 1999;27: 1350-1359.

[41] Luttringer O, Theil F-P, Poulin P, Schmitt-Hoffmann AH, Guentert TW, Lave T. Physiologically based pharmacokinetic (PBPK) modeling of disposition of epiroprim in humans. J Pharm Sci 2003;92: 1990-2007.

[42] Yang JS, Jamei M, Yeo KR, Tucker GT, Rostami-Hodjegan A. Prediction of intestinal first-pass drug metabolism. Curr Drug Metab 2007;8(7): 676-684.

[43] Sugano K. Introduction to computational oral absorption simulation. Expert Opinion Drug Metab \& Toxicol 2009;5(3): 259-293.

[44] Akimoto M, Nagahata N, Furuya A, Fukushima K, Higuchi S, Suwa T. Gastric pH profiles of beagle dogs and their use as an alternative to human testing. Eur J Pharmaceutics Biopharm 2000;49(2): 99-102.

[45] Reigner BG, Blesch KS. Estimating the starting dose for entry into humans: Principles and practice. Eur J Clin Pharmacol 2002;57: 835-845.

[46] Gueorguieva I, Aarons L, Rowland M. Diazepam pharamacokinetics from preclinical to phase I using a Bayesian population physiologically based pharmacokinetic model with informative prior distributions in WinBUGS. J Pharmacokinet Pharmacodyn 2006;33(5): 571-594.

[47] Langdon G, Gueorguieva I, Aarons L, Karlsson M. Linking preclinical and clinical whole-body physiologically based pharmacokinetic models with prior distributions in NONMEM. Eur J Clin Pharmacol 2007;63(5): 485-498. 
[48] Edginton AN, Theil FP, Schmitt W, Willmann S. Whole body physiologically-based pharmacokinetic models: their use in clinical drug development. Exp Opin Drug Metab Toxicol 2008;4(9): 1143-1152.

[49] Willmann S, Hohn K, Edginton A, Sevestre M, Solodenko J, Weiss W, Lippert J, Schmitt W. Development of a physiology-based whole-body population model for assessing the influence of individual variability on the pharmacokinetics of drugs. J Pharmacokin Pharmacodyn 2007;34(3): 401-431.

[50] Bouzom F, Walther B. Pharmacokinetic predictions in children by using the physiologically based pharmacokinetic modeling. Fundamental Clin Pharmacol 2008;22(6): 579-587.

[51] Ginsberg G, Hattis D, Sonawane B. Incorporating pharmacokinetic differences between children and adults in assessing children's risks to environmental toxicants. Toxicol Appl Pharmacol 2004;198(2): 164-183.

[52] Parrott N, Davies B, Hoffmann G, Koerner A, Lave T, Prinssen E, Theogaraj E, Singer T. Development of a physiologically based model for oseltamivir and simulation of pharmacokinetics in neonates and infants. Clin Pharmacokinet 2011;50(9): 613-623.

[53] Leong R, Vieira MLT, Zhao P, Mulugeta Y, Lee CS, Huang SM, Burckart GJ. Regulatory experience with physiologically based pharmacokinetic modeling for pediatric drug trials. Clin Pharmacol \& Ther 2012;91: 926-931.

[54] Johnson TN, Boussery K, Rowland-Yeo K, Tucker GT, Rostami-Hodjegan A. A semimechanistic model to predict the effects of liver cirrhosis on drug clearance. Clin Pharmacokinet 2010;49 (3): 189-206.

[55] European Medicines Agency. (2005). Guideline on the Evaluation of the Pharmacokinetics of Medicinal Products in Patients with Impaired Hepatic Function. Committee for Human Medicinal Products (CHMP), London, United Kingdom. 17 Feb 2005. http://www.ema.europa.eu/docs/en_GB/document_library/Scientific_guideline/ 2009/09/WC500003122.pdf.

[56] European Medicines Agency. (2012). Guideline on the Investigation of Drug Interactions. Committee for Human Medicinal Products (CHMP), London, United Kingdom. Final guideline, 21 June 2012. http://www.ema.europa.eu/docs/en_GB/ document_library/Scientific_guideline/2012/07/WC500129606.pdf. Accessed 10 Sept 2012.

[57] Food and Drug Administration. (2012). Guidance for Industry. Drug Interaction Studies- Study Design, Data Analysis, Implications for Dosing, and Labeling Recommendations. Draft Guidance, February 2012. U.S. Department of Health and Human Services, Food and Drug Administration, Center for Drug Evaluation and Research (CDER), Silver Spring, Maryland. http://www.fda.gov/downloads/Drugs/ GuidanceComplianceRegulatoryInformation/Guidances/ucm292362.pdf. Accessed 10 Sept 2012. 
[58] Zhao P, Zhang L, Grillo JA, Liu Q, Bullock JM, Moon YJ, Song P, Brar SS, Madabushi R, Wu TC, Booth BP, Rahman NA, Reynolds KS, Berglund EG, Lesko LJ, Huang SM. Applications of physiologically based pharmacokinetic (PBPK) modeling and simulation during regulatory review. Clin Pharmacol \& Ther 2011;89: 259-267.

[59] Huang S-M, Rowland M. The role of physiologically based pharmacokinetic modeling in regulatory review. Clin Pharmacol \& Ther 2012;91: 542-549.

[60] Zhao P, Rowland M, Huang S-M. Best practice in the use of physiologically based pharmacokinetic modeling and simulation to address clinical pharmacology regulatory questions. Clin Pharmacol \& Ther 2012;92: 17-20.

[61] Rowland M, Balant L, Peck C. Physiologically based pharmacokinetics in drug development and regulatory science: a workshop report (Georgetown University, Washington, DC, May 29-30, 2002). AAPS PharmSci 2004;6(1): E6.

[62] Price PS, Conolly RB, Chaisson CF, Gross EA, Young JS, Mathis ET, Tedder DR. Modeling interindividual variation in physiological factors used in PBPK models of humans. Crit Rev Toxicol 2003;33(5): 469-503.

[63] Gentry PR, Hack CE, Haber L, Maier A, Clewell III, HJ. An Approach for the Quantitative Consideration of Genetic Polymorphism Data in Chemical Risk Assessment: Examples with Warfarin and Parathion. Toxicol Sci 2002;70: 120-139.

[64] Clewell HJ, Gentry PR, Covington TR, Sarangapani R, Teeguarden JG. Evaluation of the potential impact of age- and gender-specific pharmacokinetic differences on tissue dosimetry. Toxicol Sci 2004;79: 381-393.

[65] Clewell HJ, Teeguarden J, McDonald T, Sarangapani R, Lawrence G, Covington T, Gentry PR, Shipp AM. Review and evaluation of the potential impact of age and gender-specific pharmacokinetic differences on tissue dosimetry. Crit Rev Toxicol 2002;32(5): 329-389.

[66] Gueorguieva I, Nestorov IA, Aarons L, Rowland M. Uncertainty analysis in pharmacokinetics and pharmacodynamics: application to naratriptan. Pharm Res 2005;22(10): 1614-1626.

[67] Gueorguieva II, Nestorov IA, Rowland M. Fuzzy simulation of pharmacokinetic models: case study of whole body physiologically based model of diazepam. J Pharmacokinet Pharmacodyn 2004;31(3): 185-213.

[68] Dedrick RL. Pharmacokinetic and pharmacodynamic considerations for chronic hemodialysis. Kidney Int 1975;7(Suppl 2), S7-S15.

[69] Liao KH, Tan YM, Conolly RB, Borghoff SJ, Gargas ML, Andersen ME, Clewell HJ. Bayesian estimation of pharmacokinetic and pharmacodynamic parameters in a mode-of-action-based cancer risk assessment for chloroform. Risk Anal 2007;27(6): 1535-1551. 
[70] Jorgensen WL. The many roles of computation in drug discovery. Science 2004;303(5665): 1813-1818.

[71] Ricke DO, Wang S, Cai R, Cohen D. Genomic approaches to drug discovery. Curr Opin Chem Biol 2006;10(4): 303-308.

[72] Lahoz A, Gombau L, Donato MT, Castell JV, Gomez-Lechon MJ. In vitro ADME medium/high-throughput screening in drug preclinical development. Mini Rev Med Chem 2006;6(9): 1053-1062.

[73] van de Waterbeemd H, Gifford E. ADMET in silico modelling: towards prediction paradise? Nat Rev Drug Discov 2003;2(3): 192-204.

[74] Peck CC, Barr WH, Benet LZ, Collins J, Desjardins RE, Furst DE, Harter JG, Levy G, Ludden T, Rodman JH, et al. Opportunities for integration of pharmacokinetics, pharmacodynamics, and toxicokinetics in rational drug development. J Pharm Sci 1992;81(6): 605-10.

[75] Charnick SB, Kawai R, Nedelman JR, Lemaire M, Niederberger W, Sato H. Perspectives in pharmacokinetics. Physiologically based pharmacokinetic modeling as a tool for drug development. J Pharmacokinet Biopharm 1995;23: 217-229. 
